\title{
A REVIEW OF DATA FUSION MODELS AND ARCHITECTURES: TOWARDS ENGINEERING GUIDELINES
}

\author{
Jaime Esteban ${ }^{1}$, Andrew Starr ${ }^{1}$, Robert Willetts ${ }^{1}$, Paul Hannah ${ }^{1}$, Peter Bryanston-Cross ${ }^{2}$ \\ ${ }^{1}$ The University of Manchester \\ School of Mechanical, Aerospace and Civil Engineering \\ Sackville Street, \\ Manchester, M601QD, UK \\ hitp://WwW.manchester.ac.uk \\ ${ }^{2}$ University of Warwick \\ School of Engineering, \\ University of Warwick, \\ Coventry CV4 7AL, UK
}

\begin{abstract}
This paper reviews the potential benefits that can be obtained by the implementation of data fusion in a multi-sensor environment. A thorough review of the commonly used data fusion frameworks is presented together with important factors that need to be considered during the development of an effective data fusion problem-solving strategy. A system-based approach is defined for the application of data fusion systems within engineering. Structured guidelines for users are proposed.
\end{abstract}

\section{KEYWORDS}

Data fusion, Frameworks, Intelligent systems, Engineering guidelines 


\section{INTRODUCTION}

To ensure that systems are operating within defined conditions, measurements are taken which, when analysed, enable decisions to be made based on condition. These measurements can produce data that are either very similar, often from the same sensor, or completely different from different techniques. Experienced engineers and analysts have traditionally undertaken the analysis of this data. However, with the increased computer power and development of new and novel detection systems, the data produced needs to be handled in a robust and logical manner. As such computer systems have been developed that are capable of extracting meaningful information from the recorded data. The integration of data, recorded from a multiple sensor system, together with knowledge, is known as data fusion.

Data fusion first appeared in the literature in the 1960s, as mathematical models for data manipulation. It was implemented in the US in the 1970s in the fields of robotics and defence. In 1986 the US Department of Defence established the Data Fusion Sub-Panel of the Joint Directors of Laboratories (JDL) to address some of the main issues in data fusion and chart the new field in an effort to unify the terminology and procedures. The present applications of data fusion span a wide range of areas: maintenance engineering ${ }^{[1]}$, robotics ${ }^{[2]}$, pattern recognition and radar tracking ${ }^{[3]}$, mine detection ${ }^{[4]}$ and other military applications ${ }^{[5]}$, remote sensing ${ }^{[6]}$, traffic control ${ }^{[7],[8]}$, aerospace systems ${ }^{[9]}$, law enforcement ${ }^{[10]}$, medicine, finance, metrology ${ }^{[11]}$, and geo-science.

Before undertaking a data fusion project a strategy needs to be established that can facilitate the solution of the problem in a robust and organised manner. Since the applications of data fusion are disparate, it is impossible to build a one-fits-all framework. Several generic platforms are described in the literature, which accommodate the application at hand. The 
purpose of this paper is to characterise the common ground among the various data fusion frameworks available, and underline the importance of a robust strategy to analyse the data.

Some success has been achieved in the use of neural networks for generic multi-parameter fusion, e.g. Taylor's work on Kohonen networks ${ }^{[12]}$, since the input vector, once normalised, takes in data from many sources, and the methodology fits many problems. The scope of this paper, however, is to examiner wider issues in the architecture, including frameworks, procedures and guidelines.

\section{MULTIPLE SENSOR ENVIRONMENTS}

Decisions regarding the condition of a system are seldom based upon the output of a single measurement parameter. More often, these decisions are made on the analysis of multiple parameters either from the same type of sensor or from a completely separate and different one. Thomopoulos ${ }^{[13]}$ gives a discussion regarding the advantages of multiple-sensor systems over single-sensor systems. This discussion states that there are numerous advantages in using multiple sensor systems including:

- Higher signal-to-noise ratio;

- Increased robustness and reliability in the evident of sensor failure;

- Information regarding independent features in the system can be obtained;

- Extended parameter coverage, rendering a more complete picture of the system;

- Increased dimensionality of the measurement;

- Improved resolution;

- Reduced uncertainty;

- Increased confidence; 
- Increased hypothesis discrimination with the aid of more complete information arriving from multiple sensors;

- Reduction in measurement time, and possibly costs - there is a trade off to consider in this issue. Thus, an optimal number of sensors to extract the required information from a system should be ideally pursued.

The number of sensors used is an important factor in the cost equation in terms of time, money and effort and should be limited by the information gained. Therefore, there is a need to establish a sensor performance criterion in order to assess the reliability of the measurement system selected and, at the same time be able to assign weights of evidence in the data analysis process. Richardson and $\mathrm{Mash}^{[14]}$ describe an analytical proof, which states that in most situations decisions are more reliability made when there are more rather than less sensors. However, the definition and calculation of the optimal number of sensors required for a given system is complex and one that is beyond the scope of this paper.

\section{ESTABLISHING A MULTIPLE SENSOR SYSTEM}

The actual combination of sensors is dependant upon the requirements of the system. However, a number of things need to be considered when defining the type of fusion algorithm used and level at which fusion will occur. These include:

- How are the sensors distributed?

- What are the format, type and accuracy of the collected data?

- What is the nature of the sensors used?

- What is the resolution of the sensors used?

- What is the computational capability at the sensors? 
If identical sensors are used within the multi-sensor system then the data analysis process can be done with minimal effort. This can enhance the reliability of the results provided by the redundancy of the information. On the other hand, if different types of sensors are used then the information collected needs to be formatted into a common form and aligned in the time domain.

Data can be combined either as it arrives into the system or at a defined level within the fusion process. The reliability of the data used within the fusion system will depend on the sensors available and the methodology employed for the fusion of the data. The selection of sensors as well as the number of sensors needed to increase the accuracy of the information transferred depends on the problem at hand. Different types of sensors can be used depending on the application and the output format sought. Table 1 gives a brief overview of sensors typically used in data fusion. Sensors are usually classified according to their physical nature. They are often based on the electromagnetic spectrum, sound waves, touch, odour, or the absolute position of the system. 


\begin{tabular}{|l|l|}
\hline \multicolumn{1}{|c|}{ Sensor system } & \multicolumn{1}{c|}{ Output format } \\
\hline Simple transducers & Voltage signal time series \\
\hline Infra red & Image \\
\hline Laser \& camera & Image \\
\hline Ladar & Image \\
\hline Magnetic Resonance & Image \\
\hline Optical & Image \\
\hline Particle image velocimetry (PIV) & Image \\
\hline Radar & Structured time series \\
\hline Sonar & Structured time series \\
\hline Spectroscopy & Frequency spectrum \\
\hline Ultrasonics & Structured time series \\
\hline X-Ray & Image \\
\hline
\end{tabular}

Table 1: Overview of commonly used sensor systems

\section{FRAMEWORKS FOR THE IMPLEMENTATION OF DATA FUSION SYSTEMS}

A number of data fusion frameworks have been developed both within the research and commercial environments. These frameworks have been used in numerous projects to aid the development of fusion systems by establishing the most appropriate algorithm for the defined problem.

\subsection{JDL PROCESS MODEL}

One of the most widely used frameworks is the JDL Data Fusion Framework. The Joint Directors of Laboratories (JDL) data fusion sub-panel within the US Department of Defence originally defined this system in the early years of data fusion. This framework was developed to aid the developments in military applications. Llinas and Hall ${ }^{[15]}$ describe a number of levels at which data fusion could be undertaken:

- Level 1, object refinement, attempts to locate and identify objects. For this purpose a global picture of the situation is reported by fusing the attributes of an object from multiple sources. The steps included at this stage are: Data alignment, prediction of 
entity's attributes (i.e. position, speed, type of damage, alert status, etc.), association of data to entities, and refinement of entity's identity.

- Level 2, situation assessment, attempts to construct a picture from incomplete information provided by level 1 , that is, to relate the reconstructed entity with an observed event (e.g. aircraft flying over hostile territory).

- Level 3, threat assessment, interprets the results from level 2 in terms of the possible opportunities for operation. It analyses the advantages and disadvantages of taking one course of action over another.

A process refinement, sometimes referred as Level 4, loops around these three levels to monitor performance, identify potential sources of information enhancement, and optimise allocation of sensors. Other ancillary support systems include a data management system for storage and retrieval of pre-processed data and human-computer interaction. The lay out of this model process is depicted in Figure 1. 


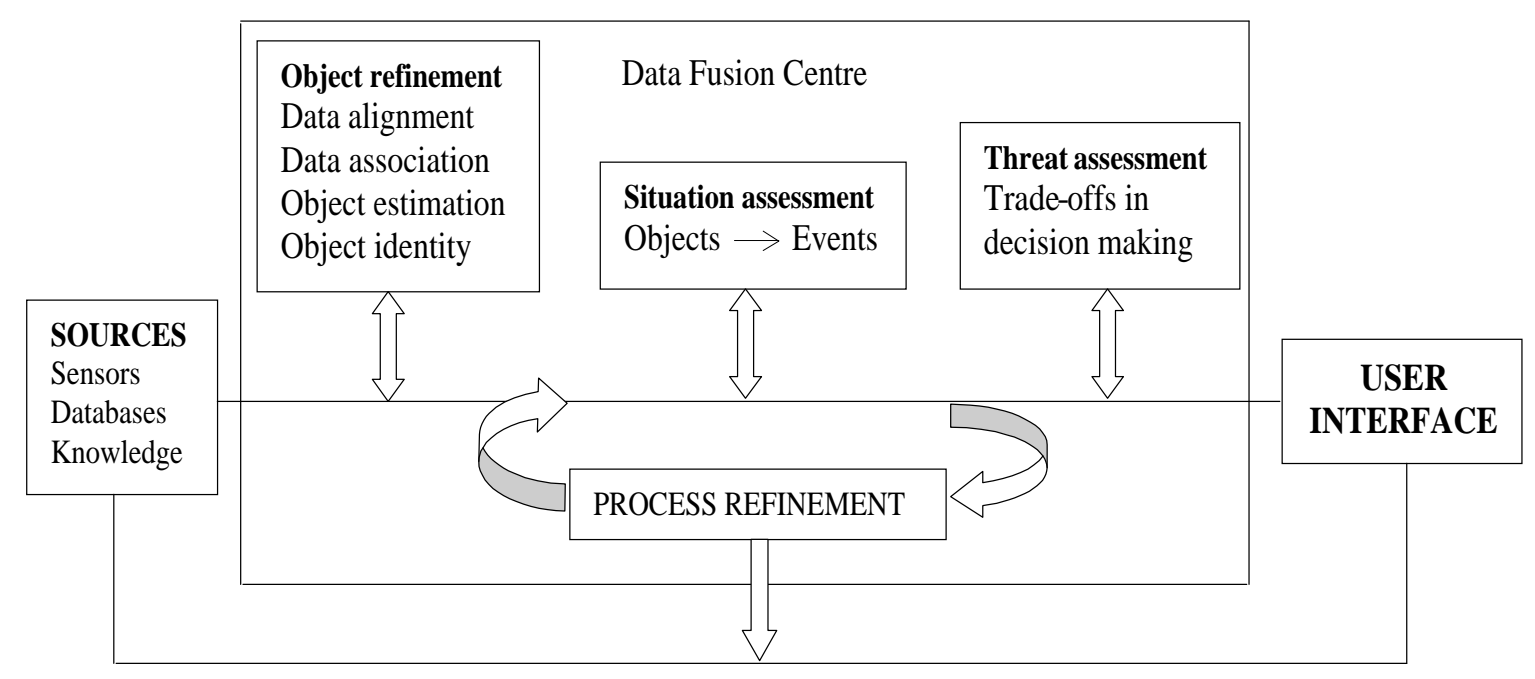

Figure 1: The JDL data fusion framework

The hierarchical distribution of the JDL model allows for the different levels to be broken down into sub-levels. In this manner, level 1 could be further divided into four processes: Data alignment, data association, object estimation, and object identity.

- At the data alignment stage, the data is processed to attain a common spatial and time frame;

- The data association could be further divided as association performed among data units of the same variable and between data units of different variables. At this stage the degree of proximity among the variables is measured;

- Object estimation, on the other hand, could be sub-divided in terms of the processing approach taken (sequential or batch), parameter identification and estimate equations available, best-fit function criteria, and the optimisation of best-fit function approach sought. At this stage the data fusion centre estimates the object's position, velocity, or attributes;

- The object identity stage could be subdivided into feature extraction, identity declaration, and combination of identity declarations. At this stage a prediction of the object's identity or classification is declared. 
At each of these lowest sub-levels, the mapping of different types of techniques could be easily allocated, and selected according to the case at hand.

Fusion can be performed on raw data in the fusion centre (centralised process) or on preprocessed locally fused data (decentralised process). A hybrid data fusion system, consisting of the integration of both raw and pre-processed data, could also be considered. The combination of the first three JDL levels into a blackboard data structure has been proposed by Paradis ${ }^{[16]}$. This framework is further integrated with a process refinement via fusion agents, which act as fusion centres.

\subsection{THOMOPOULOS ARCHITECTURE}

Thomopoulos $^{[13]}$ posed an architecture for data fusion consisting of three modules, each integrating data at different levels or modules to integrate the data, namely:

- Signal level fusion, where data correlation takes place through learning due to the lack of a mathematical model describing the phenomenon being measured.

- Evidence level fusion, where data is combined at different levels of inference based on a statistical model and the assessment required by the user (e.g. decision making or hypothesis testing).

- Dynamics level fusion, where the fusion of data is done with the aid of an existing mathematical model.

Depending upon the application, these levels of fusion can be implemented in a sequential manner or interchangeably. If continuous health monitoring of a machine is the objective, the combination of data could be done at the signal level, whilst higher order fusion (e.g. evidence fusion) would need to be applied if a wide range of decisions ought to be made from 
the signals. Figure 2 gives a summary of the architecture. Thomopoulos stressed the point that any data fusion system should consider three essential criteria to achieve the desired performance, these are:

- Monotonicity with respect to the fused information;

- Monotonicity with respect to the costs involved;

- Robustness with respect to any a-priori uncertainty.

In addition, factors such as the delay in the transmission of data, channel errors, and other communication aspects, as well as the spatial/temporal co-alignment of the data should also be taken into account in the data fusion system.

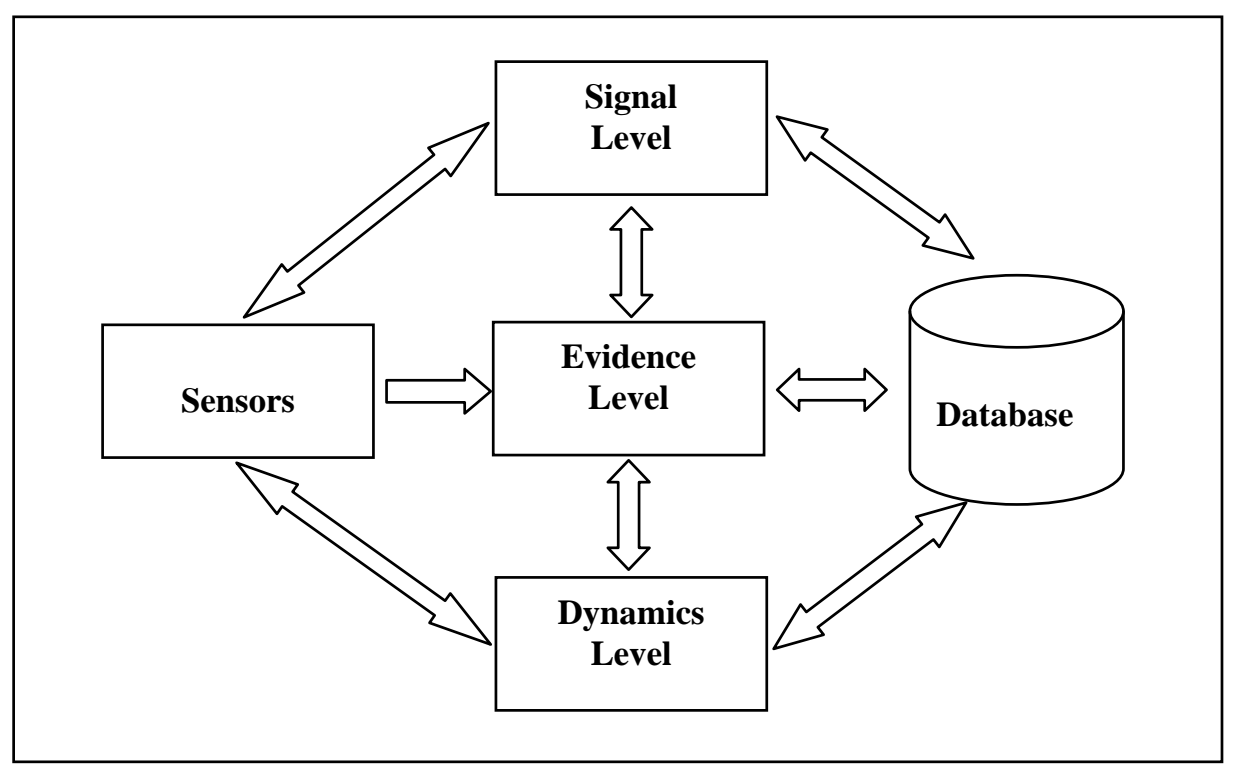

Figure 2: Thomopoulos's Data Fusion architecture

\subsection{MULTI-SENSOR INTEGRATION FUSION MODEL}

Luo and Kay ${ }^{[17]}$ introduced a generic data fusion structure based on multi-sensor integration. In this system, data from various sources was combined within embedded fusion centres in a hierarchical manner. They made a clear distinction between multi-sensor integration and multi-sensor fusion. The former refers to the use of multiple sensor information to assist in a 
particular task, whilst the latter refers to any stage in the integration process where there is an actual combination of the data.

Figure 3 shows a diagram of Luo and Kay's framework to represent multi-sensor integration and fusion simultaneously. From this diagram, the data collected at the sensor level is transferred to the fusion centres, where the fusion process takes place, in a hierarchical and sequential manner. The entire framework shown in Figure 3 is a representation of multisensor integration. A description of the measured phenomenon is obtained after the outputs of the $n$ sensors are processed, with the aid of the information system whenever appropriate. The fusion process is facilitated with the incorporation of an information system, containing relevant databases and libraries.

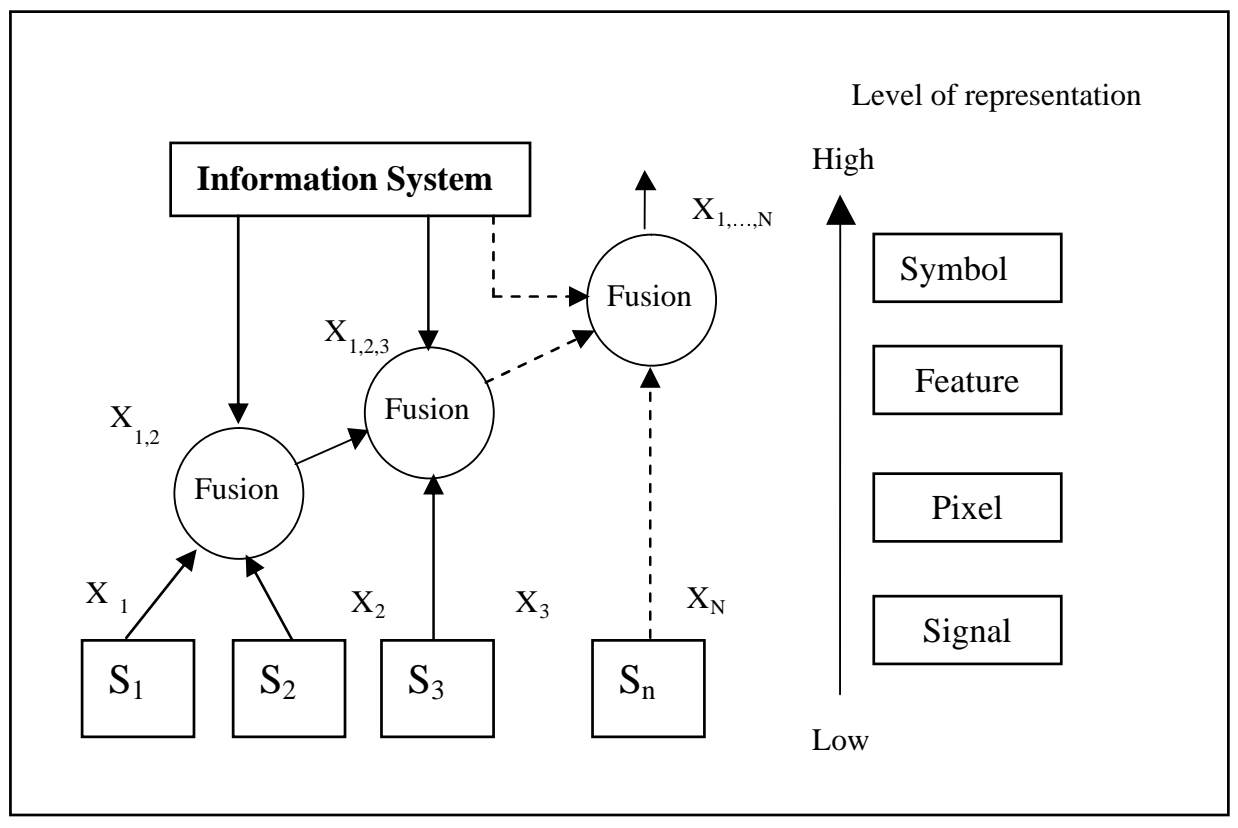

Figure 3: Luo and Kay's architecture ${ }^{[16]}$

As the information is combined at the different fusion centres, the level of representation needed is increased from the raw data or signal level to more abstract symbolic representations of the data at the symbol level. Table 2 shows a comparison of the different fusion levels classified by the representation of information. 


\begin{tabular}{l|l|l|l|l}
\multicolumn{1}{c|}{ Characteristics } & \multicolumn{1}{|c|}{ Signal level } & \multicolumn{1}{|c|}{ Pixel level } & \multicolumn{1}{|c|}{ Feature level } & \multicolumn{1}{|c}{ Symbol level } \\
\hline $\begin{array}{l}\text { Representation } \\
\text { level of } \\
\text { information }\end{array}$ & Low & Low & Medium & High \\
\hline $\begin{array}{l}\text { Type of sensory } \\
\text { information }\end{array}$ & $\begin{array}{l}\text { Multi-dimensional } \\
\text { signal }\end{array}$ & Multiple images & $\begin{array}{l}\text { Features extracted } \\
\text { from signals/images }\end{array}$ & $\begin{array}{l}\text { Decision logic from } \\
\text { signals/image }\end{array}$ \\
\hline $\begin{array}{l}\text { Model of sensory } \\
\text { information }\end{array}$ & $\begin{array}{l}\text { Random variable } \\
\text { with noise }\end{array}$ & $\begin{array}{l}\text { Random process } \\
\text { across the pixel }\end{array}$ & $\begin{array}{l}\text { Non-invariant form } \\
\text { of features }\end{array}$ & $\begin{array}{l}\text { Symbol with degree } \\
\text { of uncertainty }\end{array}$
\end{tabular}

Table 2: Characteristics of data fusion levels

\subsection{BEHAVIOURAL KNOWLEDGE BASED DATA FUSION MODEL}

$\mathrm{Pau}^{[18]}$ describes another data fusion framework based upon behavioural knowledge formalism. It can be seen from figure 4 that the framework consists of a number of basic stages that must be completed before the overall output is established. A feature vector is first extracted from the raw data. This vector is then aligned and associated to defined features. Fusion is then undertaken at the sensor attribute and data analysis levels. The final step is composed of a set of behavioural rules, which can be extracted in terms of the final representation of the fused output. Rather than assuming the blackboard architecture typically found in knowledge-based systems, this process model uses a hierarchical approach containing three levels of representation:

- The lowest level contains, for each sensor, a vector space with coordinate dimensions and measured parameters.

- The next level extracts relevant features from these vectors, and attaches labels to them.

- The third level contains a set of formalisms about the world model that relate feature vectors to events. 


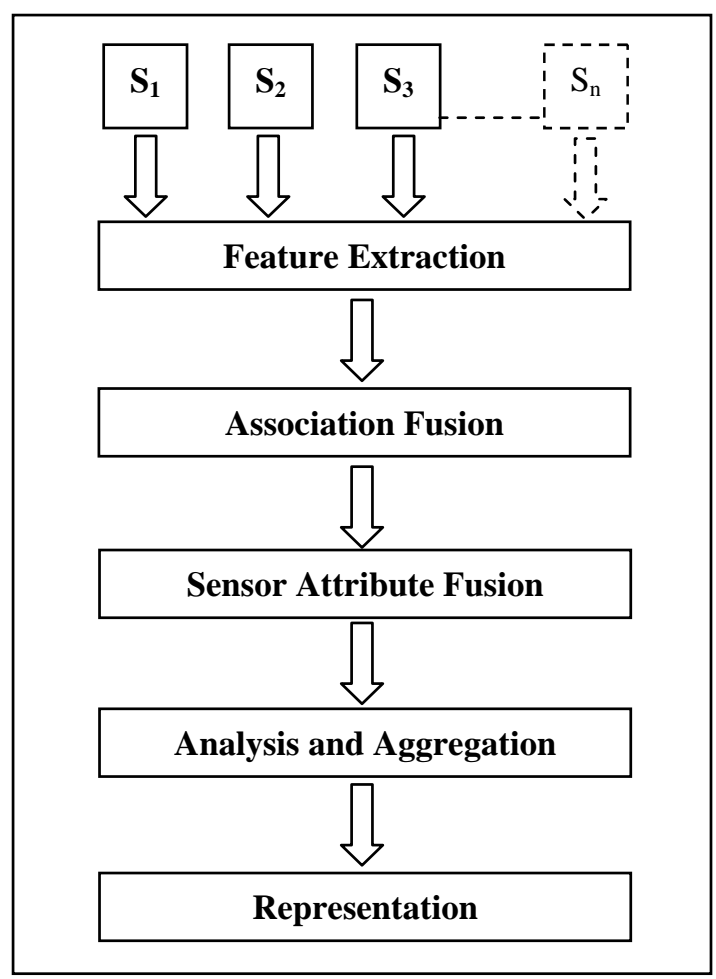

Figure 4: Pau's sensor data fusion process

Examples are given in the field of human detection and identification (e.g. intelligent buildings, security control, monitoring).

\subsection{WATERFALL MODEL}

Harris $^{[5]}$ described another example of hierarchical architecture commonly used by the data fusion community, called the waterfall model. A representation of this model is shown in figure 5. It can be seen from this figure that the flow of data operates from the data level to the decision making level. The sensor system is continuously updated with feedback information arriving from the decision-making module. The feedback element advises the multi-sensor system on re-calibration, re-configuration and data gathering aspects.

There are three levels of representation in the waterfall model, as shown in Figure 5:

- At level 1, the raw data is properly transformed to provide the required information about the environment. To achieve this task, models of the sensors and, whenever 


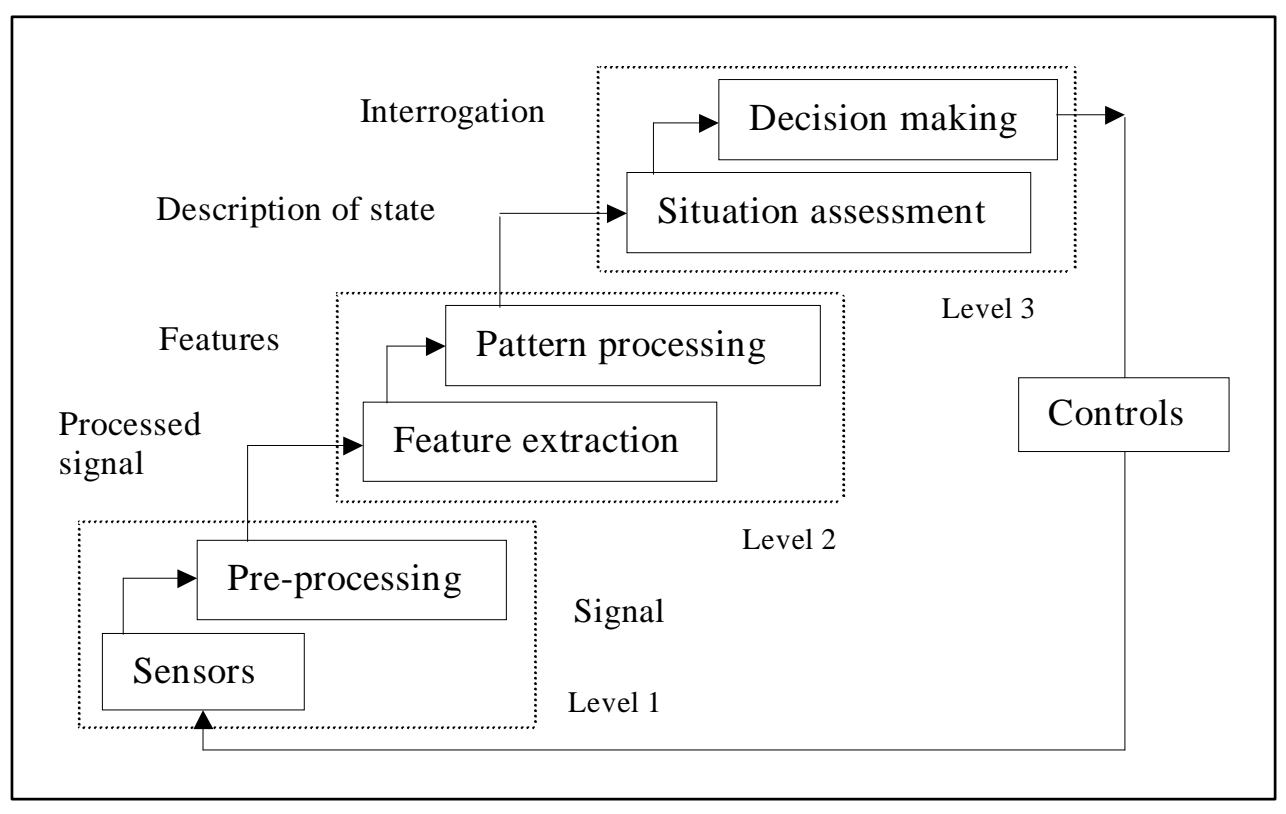

Figure 5: Waterfall model

possible, of the measured phenomena are necessary. These models could be based on experimental analysis or on physical laws;

- Level 2 is composed of feature extraction and fusion of these features. These processes are done to obtain a symbolic level of inference about the data. Their aim is to minimise the data content whilst maximising information delivered. The output of this level is a list of estimates with probabilities (and beliefs) associated with them;

- Level 3 relates objects to events. Possible routes of action are assembled according to the information that has been gathered, the libraries and databases available, and the human interaction. 


\subsection{DISTRIBUTED BLACKBOARD DATA FUSION ARCHITECTURE}

Schoess and Castore ${ }^{[9]}$ describe an example of a distributed blackboard data fusion model. This model is shown in figure 6 where 2 sensors $\left(s_{1}\right.$ and $\left.s_{2}\right)$ are connected to a number of transducers $(T)$. These sensors also have a supervisor, which controls how conflicting sensor measurements are handled. This is often based upon confidence levels assigned to each sensor. The set of transducers are used to acquire as much information as possible from the physical system under analysis (temperature, pressure, etc.). The fusion algorithm produces a value, $F$, which is dependent upon the data available to the two sensors. Confidence in the measurements is assigned to each of the sensor readings by the supervisors. This method could be defined as a database that contains sensory information and operates the communication channels available among the knowledge sources.

\subsection{OMNIBUS DATA FUSION MODEL}

Bedworth and O'Brien ${ }^{[19]}$ describe another framework called the Omnibus model. This process model is a hybrid of three other models called the Boyd loop, Dasarathy, and Waterfall models. Figure 7 shows the general layout of this framework, which consists of four main modules. These modules are used to address the various tasks in data fusion and its functional objectives. The authors describe the Boyd control loop as an iterative process with

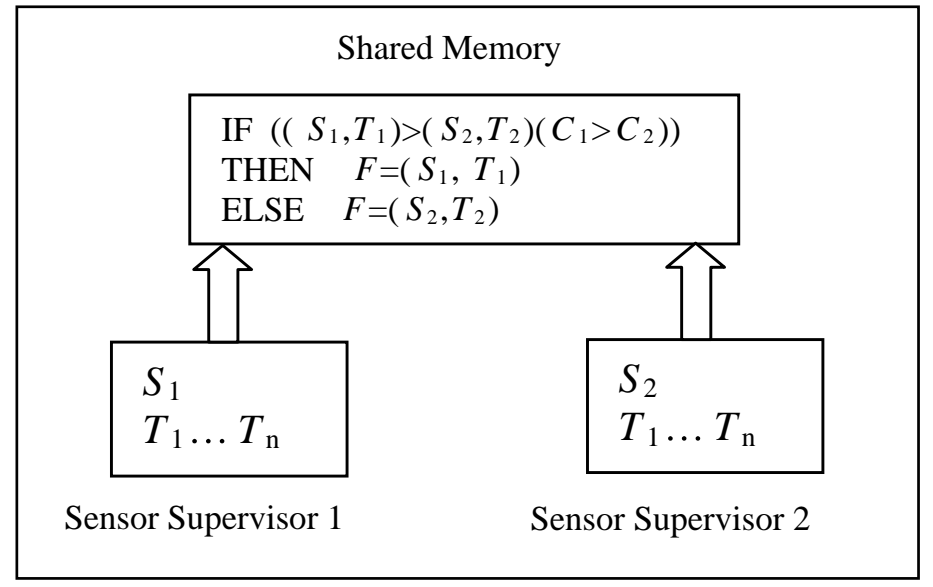

Figure 6: Distributed blackboard data fusion 


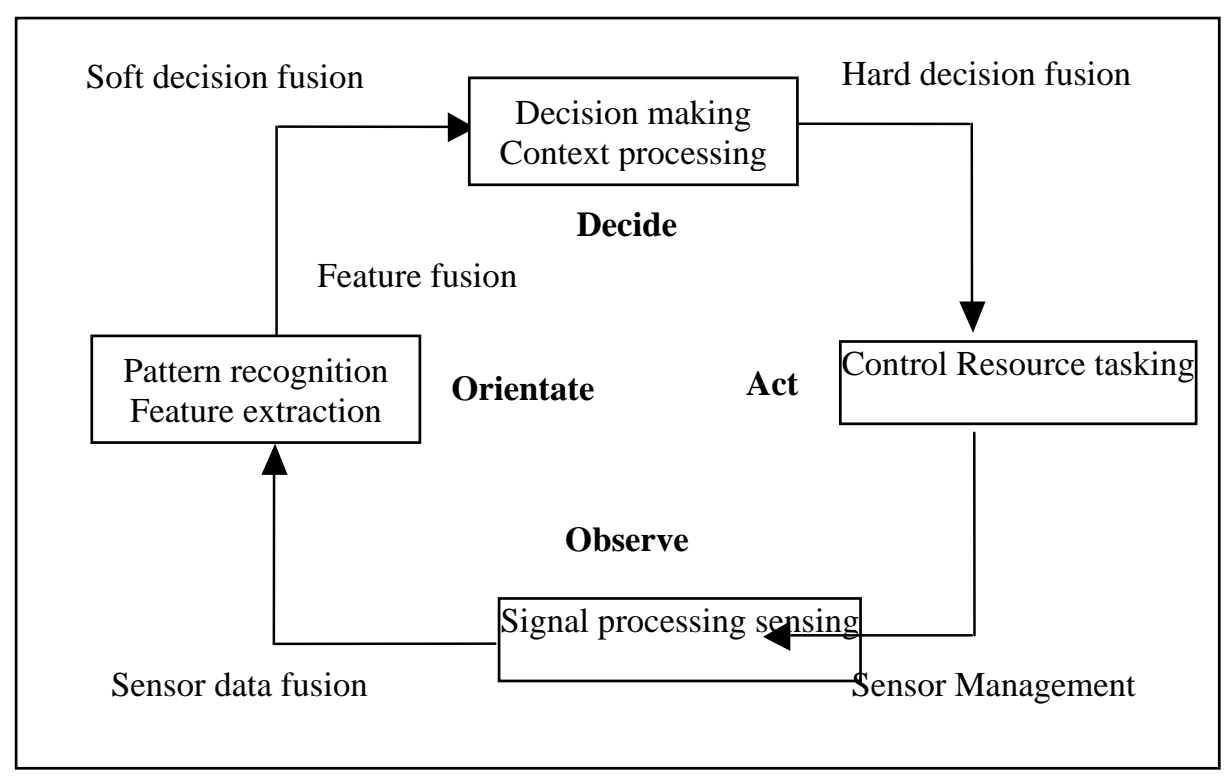

Figure 7: Omnibus data fusion model

four elements (observe, orient, decide, and act) operating in a close loop. On the other hand, the Dasarathy model consists on the three basic levels of data fusion: Data, feature, and decision.

\section{ISSUES IN THE CONSTRUCTION OF A MODEL PROCESS}

Before a robust data fusion strategy can be legitimately submitted, there is a need to underline some of the difficulties arising with the application of data fusion, as well as other features that could be incorporated into the proposed model process. Some of the difficulties arising in multi-sensor data fusion could be summarised as follows:

- Diversity of sensors used: nature, synchronisation, location, and sensor outputs.

- Diversity of data representation: image, spatial, statistical, and textual.

- Registration: the information refers to the same entity. There is a need to check the consistency of the sensor measurements ${ }^{[20]}$. This can be improved by objectively eliminating fallacious data sets.

- Calibration of the sensors when errors in the system operation occur.

- Limitations in the operability of the sensors. 


\begin{tabular}{|l|l|}
\hline \multicolumn{1}{|c|}{ Mode } & \multicolumn{1}{c|}{ Example } \\
\hline Data in-data out & Fusion of multi-spectral data \\
\hline Feature in-feature out & Fusion of image and non-image data \\
\hline Decision in-decision out & When sensors are not compatible \\
\hline Data in-feature out & Shape extraction \\
\hline Feature in-decision out & Object recognition \\
\hline Data in-decision out & Pattern recognition \\
\hline
\end{tabular}

Table 3. Data Fusion in terms of the input/output provided [23].

- Deficiencies in the statistical models of the sensors and limitations in the algorithm development.

This is by no means an exhaustive list of problems and the practitioner needs to be aware of the inherent difficulties arising in any data acquisition and data analysis tasks.

Some important architectural issues needed for the implementation of a process model for data fusion are:

- Network configuration of sensors: parallel or serial multi-sensor suite, or a combination of the two. A parallel sensor arrangement is best suited for either identical or dissimilar sensors. Serial sensor configurations are very practical when one sensor delivers complementary information to the next.

- Level of representation of the information: Although a three level system is commonly used, description of the fusion process based on input/output modes, as shown in Table 3, can aid in level selection, and adds flexibility to the JDL model ${ }^{[21]}$.

- Feedback within the data fusion network of fused information with the aid of a sensor management suite. The suite would coordinate the data, handle information flow, and store the data in a database. 


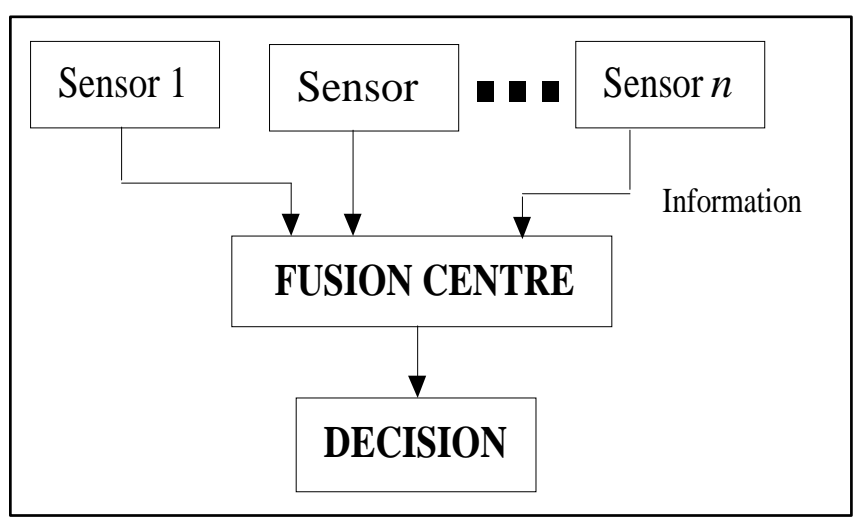

Figure 8. Centralized architecture

- The fusion of data can be done on either raw data (centralised process) or on preprocessed locally fused data (decentralised process). Hybrid data fusion, consisting of fusion of both raw and pre-processed data, can also be considered. The centralised architecture (see figure 8) is computationally intensive, but it carries the advantage of developing a global view of the object from the original data. On the other hand a decentralised architecture (c.f. figure 4) is less demanding on computational capabilities at the cost of adding complexity to the data fusion process, since each sensor has a processing unit.

- Other issues are related to these difficulties arising in data fusion, and the ability of the system to deal with them (i.e. sensor failures, corrupted data, compatibility of sensors).

Incorporation of data mining techniques to facilitate the process of data fusion should also be taken into account ${ }^{[22]}$. Data mining searches, in a systematic manner, for general relationships among data units contained in large amounts of raw data. Application of data mining techniques (clustering, neural networks, etc.) will certainly aid in the positional fusion and object identity processes.

Performance assessment is another factor that needs special consideration. Oxenham et al. described a measure of the quality of the data fusion process based on the correlated enhancement of the output information ${ }^{[23]}$. This sort of metric is determined by the 
uncertainty in the system: a decrease in uncertainty yields an increase in information delivered. Kewley gives another measure of uncertainty, provided by the system, in terms of ambiguity and vagueness ${ }^{[24]}$.

\section{A SYSTEM BASED STRATEGY: ENGINEERING GUIDELINES}

The demands for data fusion processes in a wide range of applications have made possible the proliferation of data fusion models. Providing a well-structured way to identify the system under study, regardless of the nature of the data collected, would efficiently aid in the subsequent implementation of the most convenient data fusion model. This generalised approach would allocate the necessary resources to develop data fusion into systems, rather than a data fusion system. Successful projections of particular data fusion models have been presented in the literature.

The system-based approach to data fusion proposed by the authors is shown in Figure 9. This framework was developed as part of a collaborative programme measuring flames within a harsh combustion environment ${ }^{[25]}$. It is not the authors' intention to demonstrate applications in this paper, but to examine the reasoning of the architecture. In particular, it is an attempt to meet the requirements for engineering guidelines, the need for which was emphasised by Hall $^{[26]}$. These are an important structure to link the terms of the practical problem with a multi-level algorithmic solution, which is essential to the user community.

The defined framework uses three fundamental steps in the analysis of the system. These are identification, estimation, and validation. 


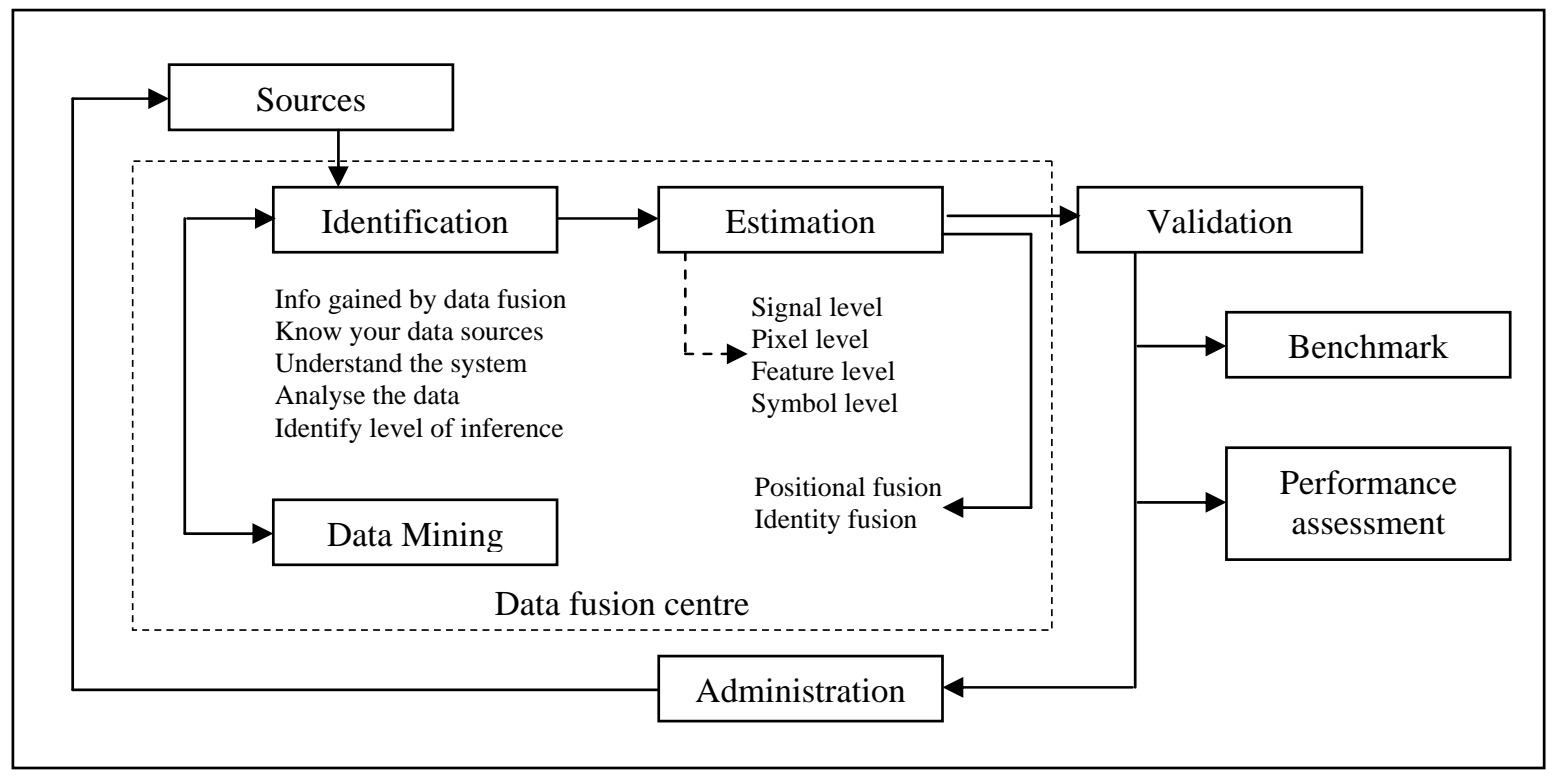

Figure 9: System-based data fusion architecture.

\subsection{IDENTIFICATION}

The identification process is aided, whenever appropriate, by the application of data mining techniques. At this stage, inference about the system takes place, interrogating the various factors used in the data fusion process:

- What is the information gained by using data fusion? This would be the first question one ought to ask before characterising a problem. It is important to identify performance criteria to identify if the data fusion process is worth doing.

- Understand the physical-chemical phenomenon under study: collect information available by fusing people's knowledge about the problem and propose a model and/or state equations describing the phenomenon. If a model already exists, it should be used and understood.

- Know all your data sources (e.g. sensors, databases, libraries). Especially, identify how the data have been collected, measuring techniques used, availability of processed data, and other issues regarding the fine tuning of the data sources, such as calibration, effects of human interaction, and missing data. 
- Analyse the data in more depth before mathematical manipulation takes place. Issues to consider include:

- checking and adjusting for the synchronisation of separate data streams;

- identifying the true dimensionality, and trying ways to reduce it, without reducing the information content;

- identifying whether the data is concentrated or sparse, and hence choosing appropriate methods for pre-processing;

- checking the repeatability of measurements, and likely error;

- examining the built-in redundancy of the sensor system to ensure a robust data collection process.

- Identify the dominant uncertainty in the system and whether this can be corrected or minimised. Uncertainty could take three forms:

- stochastic noise which cannot be corrected per se, but which could be compensated statistically;

- systematic error which might be corrected by calibration or modelling;

- unknowns, e.g. the transfer function between the real state and the measured state in a non-invasive measurement, or simply a missing parameter.

- Identify the level at which fusion must take place. Usually, data collected from similar sensors can be combined at the lowest level of inference, while data arriving from dissimilar sensors must be fused at higher levels. Fusion at a feature level, or integration of knowledge for decision-making, always occurs at a higher level.

\subsection{ESTIMATION}

Once a thorough identification of the system has been made, the process continues with the estimation of the data at the appropriate level of inference. Two taxonomies have been 
selected to select the data fusion algorithm in order to deal with a range of data sources, including time series and images:

- A four level hierarchy consisting of signal, pixel, feature, and symbol levels.

- At the signal and pixel levels of fusion, data correlation takes place due to the lack of a mathematical model describing the phenomenon being measured. The main difference between the two is the nature of the data analysed.

- At the feature level fusion, features are extracted from the raw data and then combined.

- At the symbol level fusion, the data is combined with the aid of a mathematical model and the analysis is based on statistical and logical inference.

- A classical JDL model of data fusion including positional fusion. This will attempt to determine the location and kinematic information of an entity. Following on from this is will identity the level of fusion required to transform the raw data into a meaningful representation of the system.

Both of these fusion processes complement each other, i.e. positional fusion could be exercised to facilitate the process of identity fusion, and vice versa. This taxonomy maps very closely the analytical processing needs found in the engineering community, e.g. positional fusion techniques can track down the location of the damage in a system, while identity fusion can aid in identifying the type of damage.

After the core hierarchical architecture has been selected according to the type of data and application at hand, algorithm selection is made at each levels. The information obtained at the identification stage will aid in this task.

\subsection{VALIDATION}


The processed data and the fused information is confirmed at the validation stage, where performance assessment and a benchmark procedure are implemented:

- Assessment on the performance of the data fusion model can be made measuring the uncertainty content in the solution (e.g. a probability measure, false alarm rate, or classification of accuracy);

- Build-up a benchmark procedure to improve the output results from the data fusion model, and to properly allocate the most optimal techniques.

The information gained at the validation stage can be passed onto the administration function of the model. In this manner, knowledge can be transferred and proper adjustments can be made to the model (e.g. sensor calibration, fusion techniques, measured parameters). The framework can then be most optimally mapped onto the problem at hand, leading to a sensible and methodical way to extract meaningful information from the measured phenomenon. 


\section{CONCLUSIONS}

This paper reviewed some of the most widely used architectures for the implementation of data fusion solutions to problems from different industries, for example defence, maintenance and medical. Other issues, not normally treated in detail on the framework literature, are also considered. The methodology behind successful implementation of data fusion solutions is examined in detail. The main observations are that:

- the architecture chosen is critical to successful data fusion;

- stepwise implementation including identification, estimation and validation is important for consistency, and to avoid presumptive choices;

- tailoring of the methodology, and bespoke choice of technology, based on gathered information and data from a variety of physical and human sources, is likely to lead to an optimal solution.

The authors have developed a flexible systems-based approach to data fusion which is used for a range of dissimilar sensor inputs, including time series and image arrays. The methodology allows models and information to be combined, and does not pre-determine the fusion technology. Engineering guidelines have been proposed which assist the practitioner in information gathering and decision-making. The guidelines also propose methods for validating the solutions but these need further testing.

\section{ACKNOWLEDGEMENTS}

This work was supported by the INTErSECT Faraday Partnership and EPSRC as part of project GR/M44484 “The Application of Data Fusion to a Multi Sensored Intelligent Engine". The authors gratefully acknowledge the assistance of the following partners: Corus, National Physical Laboratory, QinetiQ, Rolls-Royce, and Wolfson Maintenance; and 
particularly of Dr Mark Bedworth, Mr Graham Hesketh, Prof. John Macintyre, and Mrs Jane O'Brien in the preparation of the guidelines.

\section{REFERENCES}

1. Edwards, I., Gross, X. E., Lowden, D. W. \& Strachan, P. Fusion of NDT data. British Journal of NDT, 35 (12): 710-713.

2. Ayari, I. \& Haton, J. P. A framework for multi-sensor data fusion. Proc. IEEE Symposium on Emerging Technologies and Factory Automation 1995, 2: 51-59.

3. Linn, R. J. \& Hall, D.L. A survey of data fusion systems. Proc. SPIE Conf. on Data Structure and Target Classification, 1991: 13-36.

4. Cremer, F., den Breejes, E. \& Klamer, S. Sensor fusion for anti-personnel land mines detection. Proc. of $3^{\text {rd }}$ Eurofusion Conf. Oct. 1998: 63-70.

5. Harris C. J., Bailey A. \& Dodd, T. J. Multi-sensor data fusion in defence and aerospace. Aeronautical Journal. 102 (1015): 229-244.

6. Bruzzone, L., Fernandez, D. \& Vernazza G. Data fusion experience: from industrial visual inspection to space remote-sensing application. Proc. Academic and Industrial Cooperation in Space research, Vienna, 4-6 Nov. 1998 (ESA SP-432): 147-151.

7. Nigay, L. \& Coutaz, J. A generic platform for addressing the multimodal challenge. Proc. Conf. Human Factors in Computing Systems. 1995, 1: 98-105.

8. Sentinella, D.J. \& Raines, A. G. Real time data fusion. IEE Colloquium (Digest) 55: 5/1$5 / 3$.

9. Schoess J. \& Castore, G. A distributed sensor architecture for advanced aerospace systems. Proc. SPIE, 932, Sensor Fusion 1988: 74-86.

10. Pau, L. F. Behavioral knowledge in sensor/data fusion systems. Journal of Robotic Systems, 7 (3): 295-308. 
11. Kelly, G. Data fusion: From metrology to process measurement. NPL Internal report INTErSECT and summarised at [25].

12. Taylor, O., MacIntyre, J., 1998, Adaptive local fusion systems for novelty detection and diagnostics in condition monitoring, Proc. SPIE v 3376, p 210-218, ISSN: 0277-786X

13. Thomopoulos, S. C. Sensor integration and data fusion. Proc. SPIE 1198, Sensor Fusion II: Human and Machine Strategies, 1989: 178-191.

14. Richardson, J. M., and Mash, K. A. Fusion of multisensor data. International Journal of Robotics Research, Dec. 1988, 7(6): 78-96.

15. Llinas, J. \& Hall, D. L. An introduction to multi-sensor data fusion. Proc. IEEE International Symposium on Circuits and Systems 1998, 6: 537-540.

16. Paradis, S., Roy, J. \& Treurniet, W. 1998. Integration of all data fusion levels using a blackboard architecture. Proc. of $3^{\text {rd }}$ Eurofusion Conf. Oct. 1998: 195-202.

17. Luo, R., and Kay, M. 1988. Multisensor integration and fusion: Issues and approaches. SPIE Sensor Fusion, 931: 42-49.

18. Pau, L. F. 1988. Sensor data fusion. Journal of Intelligent and Robotic Systems 1:103116.

19. Bedworth, M. and O'Brien, J, 1999, The Omnibus model: a new model of data fusion?. Proc. 2nd Intl. Conf. on Information Fusion, Sunnyvale

20. Hackett, J. K. \& Shah, M. Multi-sensor fusion: a perspective. IEEE CH2876-1/90: 13241330.

21. Varshney, P. K. Multi-sensor data fusion. Electronics and Communication Engineering Journal Dec. 1997, 9(6): 245-253

22. Waltz, E. L. Information understanding: integrating data fusion and data mining processes. Proc. IEEE International Symposium on Circuits and Systems 1998, 6:553-556 
23. Oxenham, M. G., Kewley, D. J. \& Nelson, M. J. Performance assessment of data fusion systems. Proc. Australian Data Fusion Symp. 1996: 36-41.

24. Kewley, D. J. A model for evaluating data fusion systems. IEEE 1058-6393/93: 273-277.

\section{5. hhttp://www.eng.man.ac.uk/mech/merg/Research/Dfast/intersect.htmi}

26. Hall, D L, Garga A K, 1999, Pitfalls in data fusion (and how to avoid them), Proc. Eurofusion 99 Stratford on Avon UK 
2005-06-21

\section{A review of data fusion models and architectures: Towards engineering guidelines}

Esteban, Jaime

Springer

Esteban, J. Starr, A. Willetts, R. Hannah, P. Bryanston-Cross, P. A review of data fusion models and architectures: Towards engineering guidelines. Neural Computing \& Applications.

December 2005, Volume 14, Issue 4, pp 273-281

https://dspace.lib.cranfield.ac.uk/handle/1826/9826

Downloaded from Cranfield Library Services E-Repository 